\title{
"Neophobia": The effects of initial duration of exposure on subsequent saccharin intake in rats
}

\author{
LORNE F. PARKER \\ University of Washington, Seattle, Washington 98195
}

\begin{abstract}
Upon their first exposure to a $.02 \%$ saccharin solution, rats showed a robust "neophobic" avoidance of the solution throughout a 30-min exposure period. Furthermore, their subsequent saccharin preferences were found to be positively ralated to the duration of their initial exposure. The pertinence of these findings to conditioned taste aversions is discussed.
\end{abstract}

It has been known for some time that rats show a hesitancy toward consuming unfamiliar substances (e. g., Barnett, 1956), and, while this "neophobic" response to unfamiliar food items has been observed in both wild rats (Barnett, 1956) and to a lesser extent in domestic rats (e. g., Revusky \& Bedarf, 1967; Nachman \& Ashe, 1974), few investigations have been concerned with the ways in which "neophobia" might be affected by short-term exposures to unfamiliar edibles.

Carroll, Dinc, Levy, and Smith (1975) were perhaps the first to examine closely modifications of neophobia that occur with short-term experience. They found that domestic rats showed only a transient neophobic avoidance of a saccharin solution. The neophobic avoidance was enhanced by making the rats ill, but both their normal and illness-enhanced neophobia were found to extinguish within the first $10-20 \mathrm{~min}$ of exposure to the saccharin solution.

Domjan (1976) also found that neophobia in domestic rats was drastically attenuated by short-term experience. In contrast, however, the animals in Domjan's study still manifested neophobic avoidance toward a saccharine solution after receiving several hours of exposure to it on at least several occasions. Thus, neophobia appears to be attenuated progressively as initial exposures are extended in duration from either minutes (Carrol et al., 1975) or hours (Domjan, 1976).

Since much of the current research on conditioned and unconditioned taste preferences in rats has entailed giving rats various short-term exposures to edibles prior to conditioning treatments or preference tests, it is important to ascertain how neophobia might be affected by such short-term exposures. Thus, the purpose of the present experiment was to delineate further the effects of short-term exposures to tastes on the neophobic avoidance of such tastes. Of particular interest was the duration of exposure parameter.

The experimental design consisted first of training

Stephen C. Woods sponsors this paper and takes full editorial resəonsibility for its contents. rats to consume their daily fluid requirements within a daily 30 -min session. Various groups of rats were then offered their first exposures to a sweet saccharin solution for different amounts of time before receiving access to water for the remainder of the fluid session. Comparisons of water and saccharin consumption rates revealed the presence of neophobia during the session. The effects of initial exposure duration on neophobia were determined the following day by offering all animals access to the saccharin solution for $30 \mathrm{~min}$ and recording their rates and amounts of consumption.

\section{METHOD}

Subjects

Forty-five male Wistar rats, weighing $250-340 \mathrm{~g}$, were individually housed in stainless steel cages with ad-lib access to Purina rat pellets and $30 \mathrm{~min}$ access to tap water.

\section{Procedure}

The rats were maintained for 44 days on a daily schedule that consisted of continuous access to rat pellets and $30 \mathrm{~min}$ access to tap water beginning at 2:00 p.m. Fluid sessions consisted of attaching calibrated $50-\mathrm{ml}$ drinking tubes to the front of each cage for $30 \mathrm{~min}$. If any animal consumed less than $20 \mathrm{ml}$ of water during a session, it was given additional access to water until a total of $20 \mathrm{ml}$ of fluid intake was attained.

On Day 45 the rats were randomly divided into five groups $(\mathrm{n}=9 /$ group $)$. Group 1 received $1 \mathrm{~min}$ access to a $.02 \%(\mathrm{w} / \mathrm{v})$ sodium saccharin solution (Fisher's Scientific) at the time of the usual fluid session, followed immediately by 29 min access to water. In a similar manner, Groups $2,5,15$, and 30 received, respectively, $2,5,15$, and $30 \mathrm{~min}$ access to the saccharin solution followed by access to water for the remaining durations of the 30 -min session. Thus, all groups received a total of $30 \mathrm{~min}$ access to fluids on Day 45, but the duration of exposure to the unfamiliar saccharin solution was varied. The amount and rate of consumption of each fluid was recorded for each animal.

To assess the results of the various saccharin exposures on neophobia, all rats were given a 30-min one-bottle saccharin preference test on Day 46. Again, the amount and rate of consumption were recorded for each animal in 3-min intervals throughout the test. Food pellets were removed for $3 \mathrm{~h}$ at 30 min prior to the fluid sessions on Days 45 and 46 .

\section{RESULTS}

The rats adapted to the regimen of restricted access to water within several days, and after Day 20 virtually 


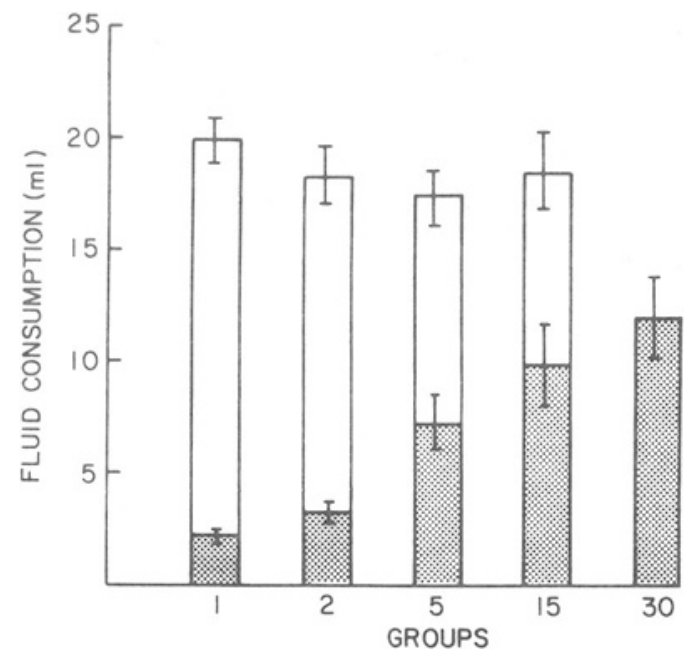

Figure 1. Mean fluid consumption of the various groups on Day 45. Saccharin and water proportions of total fluid consumption are indicated by the shaded and clear portions of the bars, respectively. Brackets indicate two standard errors of the means.

every animal consumed at least $20 \mathrm{ml}$ of water within the 30-min water sessions.

The amounts of the saccharin solution and water consumed by each group during the initial saccharin exposure session (Day 45) are shown in Figure 1. As would be expected, the amount of saccharin consumed by the various groups increased as the duration of their exposures to it was increased. These increases were not directly proportional to the duration of saccharin access, however, as the rats showed a dramatic reduction of saccharin consumption rate after the first few minutes of the session. Thus, animals in Group 30, which were not given access to water during the session, showed a significantly reduced fluid intake (see Figure 1) in spite of a commensurate opportunity to alleviate their fluid deficit $(F=3.21, \mathrm{df}=4 / 40, \mathrm{p}<.05)$.

The rats that received access to water (i.e., Groups 1 , 2,5 , and 15) all showed a significantly greater fluid intake than Group 30 on Day 45, indicating that their initial preferences for the saccharin solution were lower than their preferences for water. Since experienced rats typically prefer a $.02 \%$ saccharin solution over water (e.g., Strouthes, 1970), it appears that the rats manifested a neophobic avoidance of the saccharin solution during the session.

The effects of increasing the duration of initial saccharin exposure on the rats' subsequent saccharin preferences are depicted in Figure 2. In general, saccharin preferences were found to be enhanced by extending the durations of initial saccharin exposures from 1 to $15 \mathrm{~min}$. An analysis of variance applied to the saccharin consumption scores revealed that the effects of exposure duration on saccharin consumption were statistically significant $(F=3.74, \mathrm{df}=4 / 40, \mathrm{p}<.05)$. Individual group comparisons made with Scheffés (1953) test indicated that all pairwise comparisons were significant $(p<.05)$, with the exception of those between Groups 1 and 2, Groups 1 and 5, and Groups 15 and 30. Thus, while the rats' neophobic avoidance of saccharin persisted through even a second exposure session, the magnitude of their neophobic avoidance was significantly attenuated by extending the duration of their initial exposure to saccharin from around $5 \mathrm{~min}$ to around $15 \mathrm{~min}$.

\section{DISCUSSION}

The results of the present study are generally consistent with those reported by Carroll et al. (1975) and Domjan (1976) in that they demonstrate that the domesticated rat does manifest neophobia when confronted with a normally preferred, but unfamiliar, edible. The present results suggest, however, that the rat's neophobia is much more persistent than suggested by Carroll et al. (1975). Instead of showing a transient neophobia during only the first few minutes of their initial exposure to saccharin, the rats in the present study showed neophobic avoidance of the saccharin solution throughout the first $30-\mathrm{min}$ exposure session (Figure 1), and displayed different degrees of neophobic saccharin avoidance during a second exposure session $24 \mathrm{~h}$ later (Figure 2). Thus, the present findings are more consistent with Domjan's (1976) demonstration of neophobia persisting after several hours of exposure to a saccharin solution.

Also in agreement with Domjan's (1976) study is the finding that the degree to which rats manifest neophobia was dependent upon the duration of their previous exposures to an unfamiliar edible. The effect of exposure duration was found to be extremely robust in the present study, in that it appeared after a single exposure session and with variations of exposure durations that differed by only a few minutes. Thus, the attenuating effects of extending the duration of exposure appear to be more efficacious than reported previously (Domjan, 1976).

In conclusion, the present findings show that neophobia in the domesticated rat is more persistent than previously suggested by Carroll et al. (1975), and that it is readily modified by variation of durations of exposure from one to several minutes. In light of these findings, it would seem that the practice

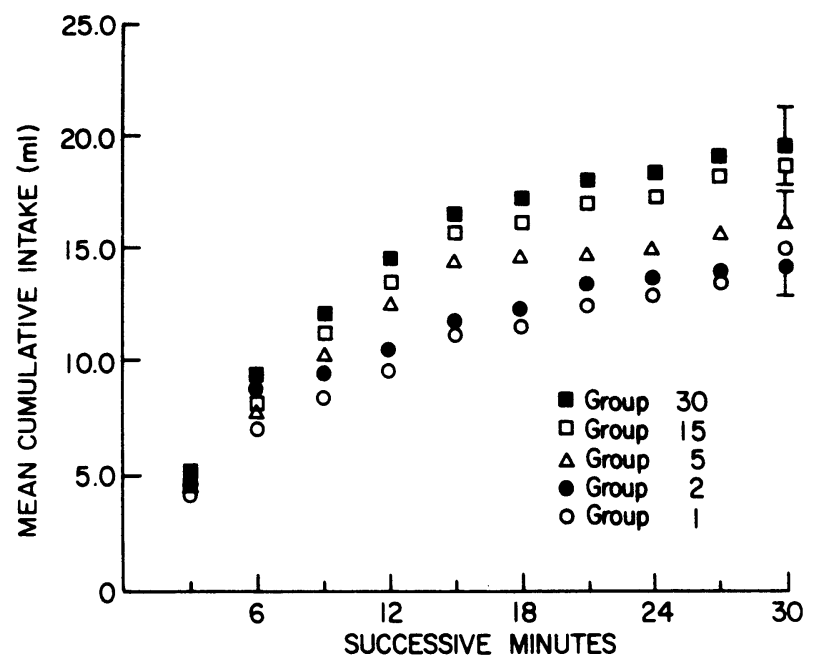

Figure 2. Mean cumulative intake of various groups during their second exposure to the saccharine solution (Day 46). Brackets indicate two standard errors of the means. 
of not controlling for the effects of exposure duration in studies of conditioned taste aversions (e. g., Bolles, Riley, \& Laskowski, 1973; Bond \& DiGiusto, 1975; Domjan \& Bowman, 1974; Kalat \& Rozin, 1973) might lead to difficulties in distinguishing between modifications of taste preferences by conditioning treatments and modifications of neophobia by differential exposures to the taste during conditioning treatments.

\section{REFERENCES}

BARNETT, S. A. Behaviour components in the feeding of wild and laboratory rats. Behaviour, 1956, 9, 24-43.

Bolles, R. C., Riley, A. L., \& Lasrowski, B. A further demonstration of the learned safety effect in food-aversion learning. Bulletin of the Pyychonomic Society, 1973, 1, 190-192.

Bond, N., \& DiGiusto, E. Amount of solution drunk is a factor in the establishment of taste aversion. Animal Learning \& Behavior, 1975, 3, 81-84.

Carroll, M. E., Dinc, H. I., Levy, C. J., \& Smith, J. C. Demonstrations of neophobia and enhanced neophobia in the albino rat. Journal of Companative and Physiological Psychology, 1975, 89, 457-467.
Domjan, M. Determinants of the enhancement of flavored-water intake by prior exposure. Journal of Experimental Psychology: Animal Behavior Processes, 1976, 2, 17-27.

Doman, M., \& Bowman, T. G. Learned safety and the CS-US delay gradient in taste-aversion learning. Learning and Motivation, 1974, 5, 409-423.

Kalat, J. W., \& Rozin, P. "Learned safety" as a mechanism in long-delay taste-aversion learning in rats. Journal of Comparative and Physiological Psychology, 1973, 83, 198-207.

NAChman, M., \& Ashe, J. H. Effects of basolateral amygdala lesions on neophobia, learned taste aversions, and sodium appetite in rats. Journal of Comparative and Physiological Psychology, 1974, 87, 622-643.

REVusKY, S. H., \& BEDARF, E. W. Association of illness with prior ingestion of novel foods. Science, 1967, 155, 219-220.

SCHEFFÉ. H. A method for judging all contrasts in the analysis of variance. Biometrika, 1953, 40, 87-104.

Strouthes, A. Long-range two-choice saccharin and water consumption in rats. Journal of Comparative and Physiological Psychology, 1970, 73, 123-134.

(Received for publication June 5, 1976.) 